\title{
Editorial on the GRG special issue on dark energy
}

\author{
George Ellis • Hermann Nicolai · Ruth Durrer • \\ Roy Maartens
}

General Relativity and Gravitation has put together a special issue on "dark energy" in cosmology, because it is a major challenge to gravitational physics and actually to all of theoretical physics. We look at the observational side (the astrophysical data for dark energy and alternative explanations of that data), phenomenological models for dark energy and possible tests of these models, and the quantum gravity side (why do we expect a very large cosmological constant? what are the possible explanations of why it is small?). The idea is not to pursue one particular approach to this important problem, but rather to produce a survey of significant approaches that have been taken as regards each of its aspects. We consider this under three major headings: ASTRONOMICAL OBSERVATIONS, GRAVITATIONAL THEORY AND PHENOMENOLOGICAL MODELS, and QUANTUM ASPECTS

Under ASTRONOMICAL OBSERVATIONS, we report on astronomical data for dark energy, which is of crucial importance: it is the first direct evidence for existence of physical fields with an effective negative gravitational energy. The crucial supernova data is surveyed by B. Leibundgut. This data is backed up by other astronomical data, surveyed by B. Nichol, and the overall observational case for dark energy is surveyed

\footnotetext{
G. Ellis (凶)

University of Cape Town, Rondebosch, South Africa

e-mail: ellis@maths.uct.ac.za

H. Nicolai

MPI for Gravitational Physics, Golm, Germany

R. Durrer

Départment de Physique Théorique, Université de Genève,

24 Quai E. Ansermet, 1211 Genève 4, Switzerland

R. Maartens

Institute of Cosmology and Gravitation, University of Portsmouth, Portsmouth PO1 2EG, UK
} 
by S. Sarkar. In interpreting the data, one must take into account the spatial curvature of Robertson-Walker models, whose effects on observations are surveyed by R. Hlozek, M. Cortês, C. Clarkson, and B. Bassett.

Under GRAVITATIONAL THEORY AND PHENOMENOLOGICAL MODELS, we have a theory overview by R. Durrer and R. Maartens, discussing also generic constraints on low-energy effective theories. Two of the main approaches are scalar fields, discussed by E. Linder, and higher order variational principles, considered by M. Francaviglia and S. Capoziello. The article by K. Koyama reviews approaches based on higher dimensional gravity and branes. Most approaches are based on the standard Robertson-Walker geometries of current cosmology, however, there are two ways this assumption might be queried: it could be that global inhomogeneities might be important as discussed by K. Enqvist, or that local inhomogeneities might be significant as discussed by T. Buchert.

The present issue also includes several articles dealing with QUANTUM ASPECTS, and more specifically with the question of whether and how dark energy and a very small cosmological constant might be understood and explained in terms of a more fundamental theory of quantum gravity. Because there is at present no generally accepted such theory, we have aimed to represent the different approaches and points of view in a hopefully balanced and informative way. After a "model independent" theoretical survey of the Cosmological Constant problem by T. Padmanabhan, the article by E. Silverstein and L. McAllister summarizes what modern superstring theory has to say about the problem, and that by R. Bousso emphasizes the explanatory power of the landscape of meta-stable vacua. Finally, the canonical side of the story is reviewed in M. Bojowald's contribution.

We hope that you will find this special issue as interesting as we do. 